\title{
Phase-inverted sidelobe-annihilated optical coherence tomography to break through the temporal diffraction limit
}

\author{
Chi Zhang ${ }^{1,2}$ and Kenneth K. Y. Wong ${ }^{2 *}$ \\ ${ }^{1}$ Wuhan National Laboratory for Optoelectronics, Huazhong University of Science and Technology, 1037 Luoyu Road, Wuhan, China \\ ${ }^{2}$ Photonic Systems Research Laboratory, Department of Electrical and Electronic Engineering, The University of Hong Kong, Hong Kong. \\ Author email address: kywong@eee.hku.hk
}

\begin{abstract}
Understand the tomography system as a temporal microscopy by space-time duality, a simple adjustment was performed on a conventional swept-source optical coherence tomography (SS-OCT), and achieved sharper resolution $(\sim 4 \mu \mathrm{m})$ for the single layer measurement.

OCIS codes: (100.6640) Superresolution; (110.4500) Optical coherence tomography; (110.6915) Time imaging.
\end{abstract}

\section{Introduction}

Optical coherence tomography (OCT) has proven to be a powerful non-invasive cross-sectional imaging modality, and it is advantageous in terms of lateral resolution, owing to its short wavelength over the ultrasound schemes [1]. Few recent breakthroughs in OCT have improved its frame rate and stability, and made it as a commercial products. However, its lateral resolution is still limited by the spectral bandwidth, namely the temporal diffraction limit. Here, we explore the analogy between the microscope and the tomography by the space-time duality [2]. As a proof-ofprinciple demonstration, a technically possible adjustment is performed on a conventional SS-OCT, and achieved sharper resolution for the single layer measurement, which is mathematically analogous to a well-known superresolution fluorescence microscopy scheme - stimulated emission depletion (STED) [3]. Therefore, understand the tomography system as a microscope in the temporal domain can leverage the ingenious super-resolution techniques in the spatial domain. It formulates a path toward super-resolution tomography with a spectrally limited bandwidth.

\section{Principle and experimental setup}

Here we take the fluorescence STED microscopy as an example: the original excited diffraction-limited PSF is compressed by introducing a doughnut shape stimulated emission depletion [3]. The most essential part is to introduce a spatial $\pi$-stepped phase mask to generate the doughnut PSF at the Fourier plane [4]. By adapting this concept to time domain, the spatial phase mask will become a temporal phase modulator, and a subtraction will replace the depletion process [2]. Apply image subtraction of a twin-peak shape from the conventional Gaussian shape, finer details can be revealed by suppressing the sidelobe of PSF. Therefore, this scheme is called as phaseinverted sidelobe-annihilated OCT (PISA-OCT).

(a)
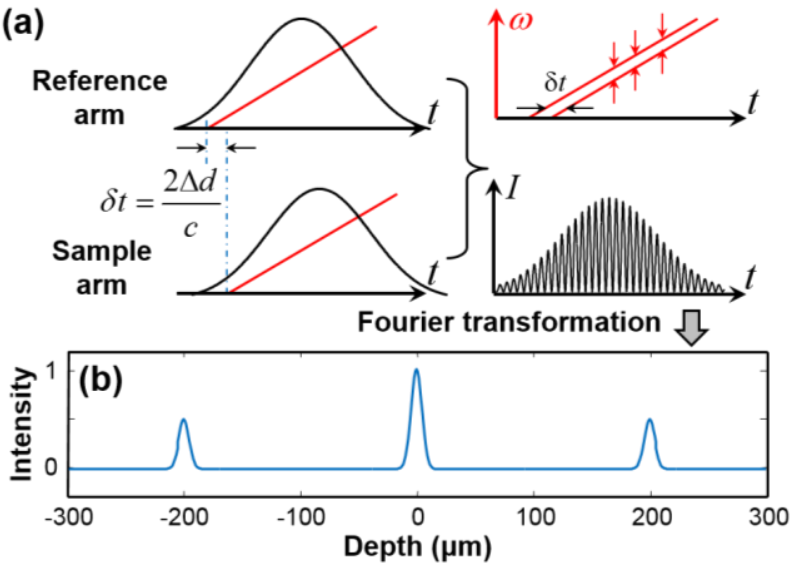

(c)
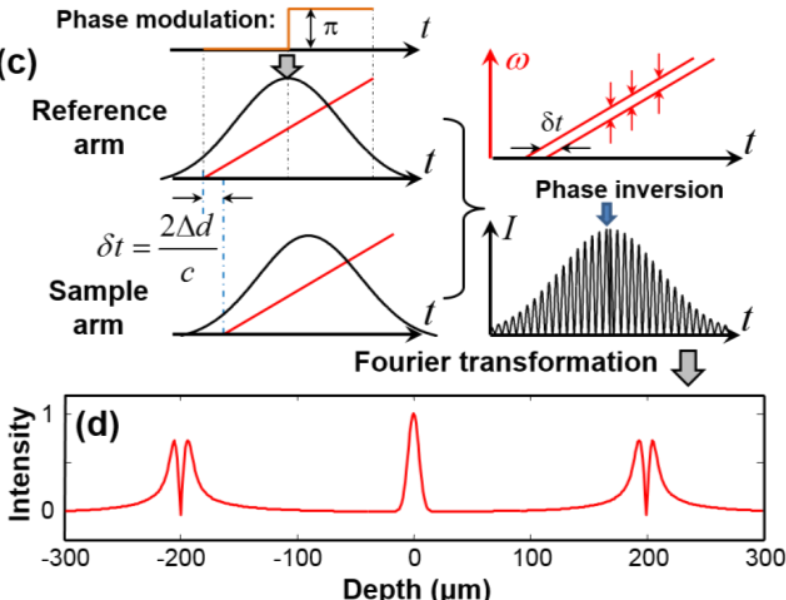

Fig. 1. Principle of the PISA-OCT. (a) \& (b), Interference and Fourier transformation process within a SS-OCT, and the Gaussian pulse is generated to reveal the depth information. (c) \& (d), The $\pi$-stepped phase modulation in the reference arm results the phase inversion after the interference, and the twin-peak shape is therefore generated. The intensity profile of the swept-source is Gaussian shape.

There are two neighboring swept-source periods considered here: the first one (Fig. 1(a) \& (b)) is a typical SS-OCT system, the spatial depth difference between the sample and reference arms of a wideband source is encoded in the interference fringes in the wavelength (or time) domain, which is required to retrieve the depth information 
(Gaussian shape) [4,5]. While the second periods (Fig. 1(c) \& (d)) are manipulated with the $\pi$-stepped phase modulation. Therefore, after the tomographic system, the interference fringes show the phase inversion in the middle, and the corresponding Fourier transformation generates the twin-peak shape PSF. Finally, the first Gaussian PSF subtracts its neighboring twin-peak shape PSF, and resultant the pulsewidth of the PSF is compressed.

\section{Results and discussions}

A stationary reflective mirror was first tested by PISA-OCT. In the ideal case, the precise frequency (namely position) is quantified by infinite long interference fringe; however, limited spectral bandwidth of the swept-source confined the fringe temporal window to be $\sim 20 \mu$ s. To quantify the observation range, the mirror was moved across the depth, to measure the roll-off curve as shown in Fig. 2(a) \& (b). Since the peak value of the PSF does not change by this subtraction, these two system sharing the almost identical roll-off curve, with 4-mm depth range. From these two figures, we also measured the pulsewidth of the PSF was compressed from $20 \mu \mathrm{m}$ to $4 \mu \mathrm{m}$ after the subtraction. To demonstrate the improved imaging quality by PISA-OCT, we obtained images from the orange juice vesicles and the onion skin. They were first measured by a SS-OCT system, the results are shown in Fig. 2(c), (e), and (g); when the $\pi$-stepped phase modulation was turned on, it became a PISA-OCT system (Fig. 2(d), (f), and (h)). It is noted that, narrower structural edging and lower background noise is achieved.
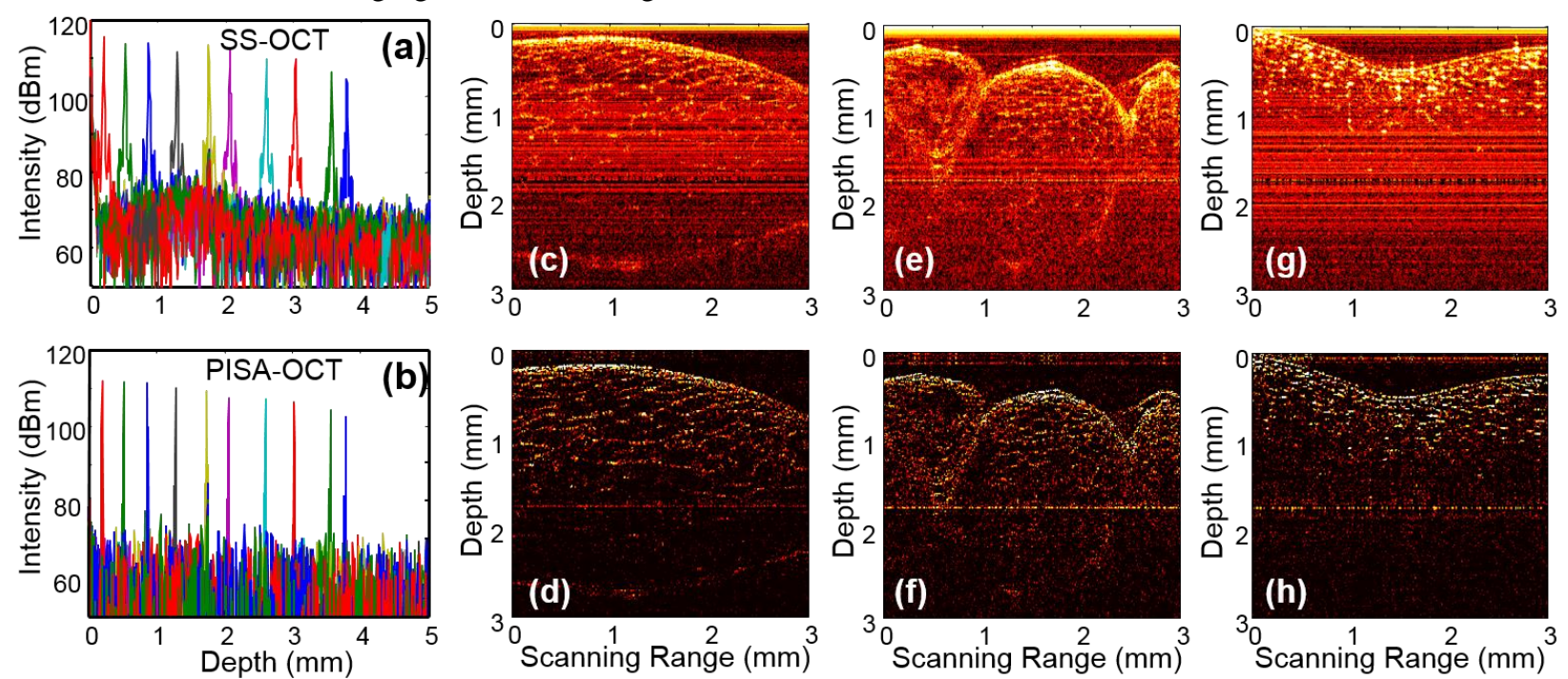

Fig. 2 Observation of the tomographic imaging improvement. Four top figures are captured by SS-OCT (with balanced detection), and the four bottom figures are captured by PISA-OCT. (a) \& (b), The roll-off measurement of the SS-OCT $(\sim 1.5 \mathrm{~dB} / \mathrm{mm})$ versus the PISA-OCT ( 1.5 $\mathrm{dB} / \mathrm{mm}$ ). (c)-(f), The cross-section feature of the orange juice vesicles. $(\mathrm{g}) \&(\mathrm{~h})$, The cross-section feature of the onion cell wall.

\section{Conclusion}

As a proof-of-principle demonstration, we here employ PISA-OCT system versus conventional SS-OCT. In addition to the resolution enhancement from $20 \mu \mathrm{m}$ to $4 \mu \mathrm{m}$, PISA-OCT also achieves four other advantages over the SSOCT: first, suppressing the DC component and the ghost fringes introduced by interlayer interference; second, improving the imaging sensitivity by lowering down the noise floor; third, electrically converted between the PISAOCT and the SS-OCT; fourth, low accuracy requirement for the phase modulation in the reference arm. Moreover, the analogy between the spatial microscopy and the temporal tomography paves an essential step to the journey of breaking the resolution limit of tomography, supersedes the preceding effort on imaging speed and stability.

Acknowledgment: The work was partially supported by grants from the Research Grants Council of the HKSAR, China (projects HKU 17208414 and HKU 717212E). The authors also acknowledge Vivolight Medical Device \& Technology Co., Ltd. for providing the SS-OCT system.

[1] D. Huang, et. al, "Optical Coherence Tomography," Science 254, 1178-1181 (1991).

[2] B. H. Kolner, "Space-Time Duality and the Theory of Temporal Imaging," IEEE J. Quantum Electron. 30, 1951-1963 (1994).

[3] S. W. Hell and J. Wichmann, "Breaking the diffraction resolution limit by stimulated emission: stimulated-emission-depletion fluorescence microscopy," Opt. Lett. 19, 780-782 (1994).

[4] Goodman, J. W. Introduction to Fourier Optics $2^{\text {nd }}$ edn (The McGraw-Hill Companies, INC. 1996).

[5] R.Huber, M. Wojtkowski, and J. G. Fujimoto, "Fourier Domain Mode Locking (FDML): A new laser operating regime and applications for optical coherence tomography," Opt. Express 14, 3225-3237 (2006). 